\title{
EVALUACIÓN DEL SISTEMA DE GESTIÓN: EL CASO DEL PROGRAMA OPCIONES PRODUCTIVAS EN SU VERTIENTE DE COFINANCIAMIENTO EN LA REGIÓN III DE YUCATÁN, MÉXICO
}

\author{
EVALUATION MANAGEMENT SYSTEM: THE CASE OF PRODUCTIVE \\ OPTIONS PROGRAM IN ITS ASPECT OF CO-FINANCING IN THE REGION III \\ OF YUCATAN, MEXICO
}

Carlos E. Leyva Morales Universidad Autónoma de Yucatán Correo: clmoral@uady.mx
Carlos Moo Novelo Universidad Autónoma de Yucatán Gobierno del estado de Yucatán Correo: carlos.moo@yucatan.gob.mx
Miguel A. Magaña Magaña Instituto Tecnológico de Conkal, Yucatán, México Correo:drmmagana@gmail.com

\section{RESUMEN}

Se evaluó la gestión y el impacto de los proyectos productivos financiados a través del programa Opciones productivas en su vertiente de cofinanciamiento en la región III de Yucatán con énfasis en la generación de empleos y mejora de las condiciones generales de bienestar de las familias. Se encontró que el programa ha tenido un éxito parcial en esta región de la entidad en la generación de empleos. Que la permanencia de los proyectos contribuyó con el 80\% del autoempleo generado entre los integrantes de los grupos apoyados. Que el programa solo está mejorando el nivel de ingreso de unos pocos de los beneficiarios del mismo en los municipios de esta región y en general no está contribuyendo a mejorar la calidad de vida, esto último ha sido resultado de otros apoyos otorgados por el gobierno federal o estatal. Y que la problemática que existe en su gestión se 
circunscribe a: excesiva demanda de solicitudes de apoyo en la Secretaria de Política Comunitaria y Social, asignación de recursos no siempre objetiva, retraso en la respuesta a los beneficiarios, el seguimiento de proyectos no es adecuado y existen conflictos de coordinación que no permiten la firma de acuerdos de colaboración entre la Secretaria de Desarrollo Social y la Secretaria de Política Comunitaria y Social.

Palabras clave: evaluación, gestión, programa y nivel de bienestar Clasificación JEL: H53, H75, I38 y J23

\section{ABSTRACT}

The administration and the impact of the productive projects funded through the Productive Options program in its aspect of co-financing in the Yucatán III region with emphasis on employment generation and improvement of the general conditions of welfare of the families were assessed. We found that the program has been partially successful in this region of the entity in the generation of jobs. That permanence of the projects contributed $80 \%$ of the self-employment generated between the members of the supported groups. The single program is improving the income level of some few of the beneficiaries of the same in the municipalities of this region and in general is not contributing to improve the quality of life, the latter is due to other support granted by the State or federal Government. The problem that exists in the program administration is limited mainly a: excessive demand of requests for support in the Ministry of community and Social policy, allocation of resources did not always objective, delay in the response to the beneficiaries, the monitoring of projects is not appropriate and there are coordination conflicts that do not allow the signing of partnership agreements between the Secretary of Social development and the Ministry of community and Social policy.

Keywords: evaluation, administration, program and welfare level. JEL Clasification: H53, H75, I38 y J23 


\section{INTRODUCCIÓN}

El análisis de la gestión de las políticas públicas es de gran importancia, ya que con él se puede determinar si los programas instrumentados cumplen con su objetivo o si realmente llega a los beneficiarios considerados. En México este proceso toma auge con la evaluación realizada al programa PROGRESA en el año 1997. Para 2007 la Secretaria de Hacienda y Crédito Público (SHCP) emite la obligatoriedad de que todo programa que ejerza presupuesto gubernamental en el país debe medir sus resultados, con indicadores tanto cuantitativos como cualitativos. Esto se debe a que, cuando el presupuesto es escaso y las necesidades son ilimitadas, se busca focalizar de manera correcta los programas (SHCP, 2007).

En el sector primario nacional,

“...el Estado mexicano y después de un largo periodo de fuertes y crecientes intervenciones en el sector agropecuario y alimenticio, inició en la década de los años 80's la adopción de medidas de reformas económicas para liberalizarlo. Los cambios se profundizaron a inicios de los años noventa, a partir de modificaciones constitucionales relacionadas con los derechos de propiedad de la tierra ejidal adoptadas en 1992, de la reducción del crédito público y del desmantelamiento de la Compañía Nacional de Subsistencias Populares (CONASUPO). El proceso de reformas culminó con la firma del Tratado de Libre Comercio de América del Norte (TLCAN) iniciado en enero de 1994 y con la eliminación del subsidio al consumo de tortilla en 1995. Con estas reformas económicas se esperaba un uso más eficiente de los recursos del campo y la transformación de la producción agropecuaria, promovida por la competencia internacional, por la seguridad en los derechos de propiedad de la tierra y por aumentos en la inversión y el crédito de la banca privada. Una expectativa adicional fue que la liberalización del campo aceleraría en el corto y mediano plazo la emigración rural al resto de México y a Estados Unidos..” (Yúnez, 2010).

En paralelo a estas reformas, el Estado mexicano aplicó políticas públicas cuyo objetivo era que el agro transitara a un contexto de libre mercado. Destacan Apoyos y Servicios a la Comercialización Agropecuaria (ASERCA), creada en 1991, el Programa de transferencias directas de ingreso a agricultores o Procampo y los subsidios a los productores 
comerciales y procesadores privados, también llamado "Ingreso Objetivo"; ambas acciones para el sub-sector de cultivos básicos. "La cobertura de Procampo ha sido amplia, beneficiando a más de 2.5 millones de agricultores en promedio anual en todo el país y en cuanto a los agentes que ha apoyado Ingreso Objetivo, no han rebasado los 100 mil al año y sus apoyos han privilegiado a productores comerciales de maíz, trigo, algodón y sorgo del norte del país" (Yúnez, 2010).

Por último, en 1995 el Estado creó Alianza para el Campo con el propósito general de relacionar a los productores rurales con la cadena alimenticia, mismo que incluía a los situados en áreas marginadas del país (Yúnez, 2010). Sin embargo, las características de las políticas públicas al campo durante las reformas señaladas dieron lugar, y explican al menos en parte, a la ausencia de cambios sustanciales en la estructura agropecuaria del país durante ese periodo. Entre ellas destaca el privilegio que las políticas de gasto gubernamental ha dado a la provisión de bienes privados — no públicos como las obras de infraestructura y la investigación y desarrollo-, así como su carácter regresivo. Respecto a esto último, hay evidencia de que $10 \%$ de los productores agrícolas con más tierra reciben entre 50 y $80 \%$ de los subsidios al sector, por ejemplo, el subprograma Ingreso Objetivo es el más regresivo dado que $80 \%$ de su presupuesto se ha canalizado a este tipo de propietarios (Scott, 2006; Taylor et al., 2007).

En el sexenio de Calderón Hinojosa se instrumentaron los siguientes programas para impulsar el desarrollo rural: Programa de atención a contingencias climatológicas (PACC), Programa soporte (asistencia técnica y capacitación), Proyecto regional de asistencia técnica al microfinanciamiento rural (PATMIR) y Adquisición de activos fijos (Alianza para el campo). Todos ellos buscan mejorar las condiciones de vida de las personas dedicadas a las actividades productivas del campo; sin embargo, muchos de sus recursos no están llegando a quienes lo necesitan, ya sea por malos manejos por parte de funcionarios o porque los productores no conocen los mecanismos para solicitarlos. De ahí que, por más que se esté invirtiendo en el campo, no se vean resultados palpables en la reducción de la pobreza patrimonial de los campesinos.

Dado el contexto anterior, el presente trabajo tiene la finalidad de evaluar como la gestión de los proyectos productivos financiados a través 
del programa Opciones productivas (OP), en su vertiente de cofinanciamiento en la región III de Yucatán, impactó en el empleo (autoempleo y nuevo empleo) en las comunidades donde se desarrolló y si contribuyó a mejorar los niveles de bienestar (ingreso, educación, vivienda, electrodomésticos y salud) de las familias de la región. El análisis está estructurado de la siguiente manera: en la segunda sección se presenta el marco teórico-empírico donde se describen los encuadres teóricos considerados, así como el estado del arte en relación con el fenómeno estudiado; en la tercera se desarrolla la metodología en que se baso su realización (diseño de comprobación, técnicas, instrumentos y forma de procesamiento). La cuarta sección contiene los resultados obtenidos, divididos en cinco secciones: programa Opciones productivas en la región III de Yucatán, características de los proyectos productivos financiados por OP, indicadores del impacto del programa, características de la gestión del programa OP e indicadores de eficacia su gestión. El trabajo concluye con la presentación de los juicios finales que argumentan el logro del objetivo planteado y recomendaciones.

\section{MARCO TEÓRICO}

Se tomó en cuenta el encuadre teórico sobre la evaluación del sistema de gestión de programas propuesto por Osuna y Márquez (2005), quienes establecen que para la evaluación de la gestión de programas públicos se debe utilizar el enfoque integral de gestión del ciclo de vida de un proyecto, el cual comprende las fases de programación de las actuaciones, de diseño de los mecanismos de ejecución y gestión, la ejecución y gestión del programa y la evaluación. Se examinan todos los aspectos de gestión relacionados con cada una de estas fases, fundamentalmente la división de tareas y competencias, la coordinación establecida entre los distintos organismos responsables de los programas, la suficiencia de medios económicos y humanos para una gestión correcta y eficaz.

Las otras aproximaciones teóricas consideradas en el estudio fueron:

1) Teoría de la participación burocrática y de la organización. En ésta se identifican las variables que pueden ocasionar que fracase la 
instrumentación de un programa, el proceso de comunicación, las capacidades y las actitudes hacia la política, de esta forma se considera que los asuntos burocráticos son la causa principal del fracaso de la indtrumentación de los programas o políticas (Kaufman, 1973).

2) El enfoque Top-Down. Este inicia con el análisis de la toma de decisiones, usualmente un estatuto, para después examinar el grado en que los objetivos de un programa, legalmente ordenados, fueron alcanzados en el tiempo y por qué; para ello analiza el comportamiento de los burócratas y de los grupos objetivo hacia la decisión política, el logro de objetivos a través del tiempo, los factores principales que afectan los impactos de la política y sus resultados y la reformulación de la política (Elmore, 1978).

3) Teoría del empleo de Keynes. Aquí se argumenta la participación del Estado como agente que puede impulsar la inversión en los sectores pobres y en las áreas donde las empresas privadas no invierten, y, al mismo tiempo, considera que la generación de empleo es importante para mejorar la calidad de vida de las personas (Keynes, 1972).

4) Teorías del desarrollo. En ellas se señalan como factor de cambio el que la población acceda a mejor educación, alimentación, salud y vivienda (So, 1990).

5) Teorías de la pobreza. Consideran si los proyectos están generando los suficientes ingresos para sacar a los beneficiarios de la línea de la pobreza y, al mismo tiempo, si realmente esos proyectos son detonadores para que esas personas mejoren sus condiciones de vida (Altimir, 2003).

Los encuadres anteriores permitieron complementar el análisis de las relaciones entre las variables consideradas en el trabajo: los apoyos del programa Opciones productivas, el empleo y los niveles de bienestar.

Por otra parte, es de importancia señalar que entre los estudios más recientes realizados sobre el tema tratado está el de Lacalle, Rico y Durán (2008), quienes evaluaron el programa de microcréditos de Cruz Roja Española en Ruanda, encontraron que las familias participantes en el programa de microcréditos poseen un mayor nivel de bienestar económico y que, además, existen indicios de que la concesión de microcréditos incrementa la capacidad productiva de las familias y su estatus social. Un segundo estudio es el de Martínez (2007), el cual con base en 
los procedimientos establecidos en la herramienta llamada análisis organizacional y de programas evalúa el programa Chile Barrio desde el punto de vista de la gestión institucional, en él concluye que el programa constituyó una propuesta innovadora en materia de política social y que el balance global muestra aspectos positivos como mayor diversidad y completitud en la provisión de soluciones (comparado con los programas regulares del Ministerio de Vivienda y Urbanismo, focalización territorial efectiva, una adecuada información y participación de los pobladores). Los aspectos negativos fueron la coordinación intersectorial poco efectiva, existencia de inequidades horizontales y la falta de relevancia del control de gestión. Por último, se encuentra el trabajo de Campos y Pascuali (2010), quienes evaluaron la gestión de programas de reciclaje en escuelas de educación básica y concluyeron que, aunque hay una organización eficaz, no siempre resulta eficiente y la relación de las instituciones con los gestores externos del material reciclable representa el aspecto más problemático — la justificación y objetivos del reciclaje no concuerdan, sustituyéndose por metas asociadas a logros materiales - y que el personal no ha interiorizado el elemento conservacionista del reciclaje, aunque los estudiantes sí.

\section{METODOLOGÍA}

El trabajo se basó en un diseño de comprobación de hipótesis del tipo no experimental transversal, en su variante correlacional (Hernández, Fernández y Baptista, 2010). La información de interés se recopiló utilizando la técnica de revisión de literatura, que incluyó estudios anteriores de programas similares, así como diversas fuentes de información de las instituciones relacionadas con la temática (evaluaciones del Consejo Nacional de Evaluación y de la Secretaría de Política Comunitaria y Social).

En la recopilación de la información documental se utilizaron fichas de trabajo para concentrar y resumir la información contenida en las fuentes consultadas, así como la que se obtuvo del trabajo preliminar de campo o de reconocimiento de la zona objeto de estudio. El material recopilado se ordenó y clasificó en función de las variables e indicadores que fueron de relevancia para el estudio. 
Lo anterior se complementó con la aplicación de una encuesta por muestreo aleatorio simple (realizada en el año 2009) que utilizó la fórmula siguiente para determinar el número de proyectos a considerar en la muestra:

$$
\begin{gathered}
\mathrm{n} 0=(z 2 \alpha / 2)(p)(q) / d 2 \\
\mathrm{n}=\frac{n 0}{1}+\frac{n 0}{N}
\end{gathered}
$$

Dónde:

$(\mathrm{Z} 2 \alpha / 2)$ coeficiente que se obtiene cuando la confianza o certeza de ocurrencia del evento es de $95 \%, Z$ toma el valor de 1.96 y se eleva al cuadrado; $p$ es la proporción de éxito de la variable; $q$. es la proporción de fracaso de la variable; $d 2$ es el error elevado al cuadro; $N$ es el número de unidades muestrales en la población; y $n$ es el número de unidades muestrales seleccionados en la muestra. El tamaño de muestra resultante fue $n=93.75$

Los 94 proyectos se seleccionaron del padrón de beneficiarios que lleva a cabo la Secretaría de Política Comunitaría y Social, y no todos los proyectos estudiados continuaron en operación. La cedula de entrevista para beneficiarios constó de un total de 31 preguntas, 29 cerradas y dos de tipo abierto. La información que recopiló fue sobre el empleo generado, la permanencia de los proyectos productivos y el nivel de vida de los beneficiarios con el programa OP del año 2003 al 2007 (cuadro 1).

\section{Cuadro 1}

Indicadores de impacto del Programa

\begin{tabular}{|l|l|}
\hline \multicolumn{1}{|c|}{ Indicador } & \multicolumn{1}{c|}{ Valor } \\
\hline $\begin{array}{l}\text { 1. La generación de empleo y de } \\
\text { autoempleo }\end{array}$ & $\begin{array}{l}\text { Número de empleos nuevos permanentes o } \\
\text { temporales creados. }\end{array}$ \\
\hline 2. La permanencia del proyecto & Dos años o más de operación \\
\hline $\begin{array}{l}\text { 3. Elnivelde vida de los beneficiarios } \\
\text { del crédito }\end{array}$ & $\begin{array}{l}\text { Crecimiento del ingreso anual, nivel educativo, } \\
\text { las condiciones de la vivienda, el tipo de } \\
\text { alimentación, la compra de electrodomésticos } \\
\text { y acceso a servicios de salud. }\end{array}$ \\
\hline
\end{tabular}


Se aplicaron también entrevistas a informantes clave, estos fueron los responsables del programa (coordinadores), a quienes se les administró una guía de entrevista de 15 preguntas que cuestionó sobre aciertos y problemas del programa en su gestión y seguimiento, áreas de oportunidad para modificar, porcentaje de solicitudes atendidas, medios de difusión y promoción del programa y si OP está generando empleos permanentes en las comunidades con alto grado de marginación.

La medición de la gestión del programa se realizó con base en cinco características que fueron:

1. Tiempo de ingreso y recepción del financiamiento,

2. Operación y administración del proyecto,

3. Visitas de seguimiento al proyecto y capacitación a los grupos beneficiarios,

4. Dificultades en cuanto al seguimiento del proyecto,

5. Otros apoyos otorgados por el gobierno federal.

En tanto que los indicadores de eficacia del Programa que se consideraron en el trabajo fueron:

1. Tiempo de respuesta a la solicitud del beneficiario,

2. Coordinación entre los involucrados en la administración y ejecución del programa,

3. Medios de promoción y difusión del programa,

4. Cumplimiento de los objetivos planteados en el programa.

La información se codificó y registró en cuadros de Excel y se realizó un procesamiento estadístico de los datos (cálculo de valores promedio y porcentajes). En tanto que el análisis de los resultados se basó principalmente en los principios teóricos de la evaluación del sistema de gestión de programas propuesto propuestos por Osuna y Márquez (2005). 


\section{RESULTADOS}

\subsection{Programa Opciones productivas en la región III de Yucatán}

El programa Oportunidades productivas tiene como objetivo apoyar a la población que vive en condiciones de extrema pobreza, en forma individual o en grupo están interesados en la creación, reforzamiento o ampliación de proyectos productivos. También busca fomentar el incremento de su patrimonio productivo o capital (Coneval, 2007).

De 2003 a 2007 en el estado de Yucatán se apoyaron bajo este programa 1003 proyectos productivos de diversas actividades y se beneficiaron 7632 personas; 3652 hombres y 3980 mujeres, con una inversión de 82.73 millones de pesos. Actualmente las modalidades de Opciones productivas son: agencias de desarrollo local, red de mentores, proyectos integradores y fondo de cofinanciamiento.

La última vertiente del programa, fondo de cofinanciamiento, consiste en apoyos recuperables para proyectos productivos, unidades familiares, grupos sociales y organizaciones de productoras y productores en condiciones de pobreza patrimonial que se desarrollen en la zona de cobertura (DOF, 2010). En la región III del estado el total de proyectos financiados por este programa fue de 124 en el periodo 2005-2009, con una inversión total de 7.4 millones de pesos para beneficio de 713 personas, tanto en grupos productivos como de manera individual.

\subsection{Características de los proyectos productivos financiados por OP en la región III}

De los grupos financiados por el programa, $73 \%$ se enteró de su existencia a través del ayuntamiento, en tanto que $25 \%$ lo hizo mediante un amigo o familiar. Así mismo, de los representantes de cada grupo beneficiado 94\% manifestó que el financiamiento recibido lo utilizó para capital de trabajo, es decir, para adquirir insumos para su actividad productiva, en tanto que sólo 5\% lo destinó para adecuar o construir las áreas para desarrollar su proyecto productivo. 
Por otra parte, del total de proyectos productivos sólo en $52.68 \%$ de ellos la actividad que se le financió continúa en operación (49 proyectos), mientras que el $47.31 \%$ restante han dejado la actividad que les fue apoyada. De los proyectos financiados en los municipios de la región que continúan en operación, $20.40 \%$ son financiamientos que fueron otorgados durante el año 2006, 40.81\% provienen de apoyos recibidos en 2007 y $38.79 \%$ son actividades a las que se les brindó el apoyo en el año 2008.

De los proyectos que actualmente continúan en operación en la región III del estado se pudo observar que la actividad a la cual se le destinó el mayor recurso, y que todavía permanece en operación, fue la producción de ovinos con un millón 23 mil 352 pesos; en su inició benefició a 70 personas, seguido en importancia por la engorda de porcinos a la que se le destinó 470.9 mil pesos en beneficio de 31 personas. En contraste, la actividad productiva a la cual se le destino el menor recurso, y que permanece en operación, fue a una lavandería que recibió un apoyo de 15 mil pesos y benefició a solo dos personas.

En cuanto al número total de proyectos apoyados que continúan en operación por tipo de actividad, se pudo apreciar que la cría de ovinos es la actividad con mas proyectos (11 en total), seguida de la engorda de porcinos con 10 proyectos financiados; de menor importancia están los apoyos a la carpintería y al urdido de hamacas, con cuatro proyectos cada uno de ellos.

En lo que concierne a los montos de inversión en los municipios de la región III donde los proyectos del programa Opciones productivas permanecen en operación, se puede observar en el cuadro 2 que fue Tepakan el que recibió el mayor monto (inversión total de 993.9 mil pesos), le siguió en importancia el de Izamal (593.5 mil pesos), en contraste en el municipio de Hoctún únicamente se invirtió un total de 93.5 mil pesos en los proyectos del programa. 
Cuadro 2

Inversión por municipio en la región III

\begin{tabular}{|l|c|}
\hline \multicolumn{2}{|l|}{} \\
\hline Municipio & Inversion \\
\hline Tepakan & 993890 \\
\hline Izamal & 593500 \\
\hline Tekanto & 439714 \\
\hline Hocaba & 393500 \\
\hline Sanahcat & 361000 \\
\hline Tekal de venegas & 184000 \\
\hline Teya & 175941 \\
\hline Cuzama & 147000 \\
\hline Hoctun & 93500 \\
\hline TOTAL & $\mathbf{3 ~ 3 8 2 ~ 0 4 5}$ \\
\hline
\end{tabular}

Fuente: Elaboración propia con base en los resultados de la entrevista a productores.

Es importante señalar que del total de proyectos que permanecen en operación del programa en la región, $51 \%$ sí recibieron capacitación en el desarrollo de su proyecto productivo, mientras que $49 \%$ no lo recibió para la operación y puesta en marcha de sus proyectos. Los principales temas sobre los que recibieron capacitación fueron: cría y engorda de animales, manejo de apiarios, manejo de máquinas de bordar y temas relacionados con la contabilidad. Lo anterior hace evidente que este es un factor que puede contribuir a incrementar la permanencia de más proyectos productivos bajo el programa, siempre y cuando se proporcione de manera periódica, y no solo una vez como sucedió en los proyectos apoyados en esta región de la entidad.

En lo que respecta a los grupos que se conformaron para solicitar el crédito, $55.1 \%$ estaba integrado por grupos de una a cinco personas, $38.8 \%$ de seis a diez personas y solamente $6.1 \%$ se integró por más de diez personas. Esto da idea de que los proyectos financiados en esta región se integraron en su mayoría por familiares, y que en general fueron proyectos productivos de baja inversión. 


\subsection{Indicadores del impacto del programa OP en la región III de Yucatán}

Los indicadores del impacto del programa OP evidenciaron que los proyectos sirvieron para generar autoempleo en las mismas comunidades y entre los beneficiarios del grupo, ya que $80 \%$ de los proyectos que continúan en funcionamiento generó autoempleo entre los familiares de los beneficiarios ( $\sin$ seguridad social) y sólo en $20 \%$ se requirió para su operación la contratación de nuevo personal (empleo formal sin seguridad social).

Por otra parte, en cuanto al empleo de nuevo personal se tiene que, en ocho proyectos productivos, los beneficiarios contrataron de una a dos personas para trabajar en la actividad apoyada, mientras que sólo en dos de ellos contrataron de tres a cinco personas. Esto, evidentemente, muestra que el programa OP si está cumpliendo con el objetivo de crear empleo y autoempleo en las comunidades de la región, aunque en menor proporción de la que se esperaba, lo que contribuye a reducir, en alguna medida, la migración de las personas de estas municipalidades hacia otros lugares en busca de oportunidades de trabajo. Sin embargo, falta mucho por avanzar al respecto en este programa, ya que, como se indicó en párrafos anteriores, apenas $52.7 \%$ de los proyectos financiados continúan en operación, el impacto de OP pudo haber sido mucho mayor en materia de empleo y autoempleo en los municipios beneficiados.

Es importante señalar que en $57 \%$ de los proyectos productivos apoyados en total por OP participaron de una a cinco personas (autoempleadas o contratadas), mientras que en $36.7 \%$ lo hicieron de seis a diez personas y sólo en $6.12 \%$ de los casos se registra una participación de 11 a 15 personas (gráfica 1). El bajo porcentaje del segundo grupo de personas (6 a 10) se explica por los efectos de la crisis económica de los años 2007 y 2008 en el contexto nacional. 


\section{Grafica 1}

Personas empleadas en los proyectos productivos

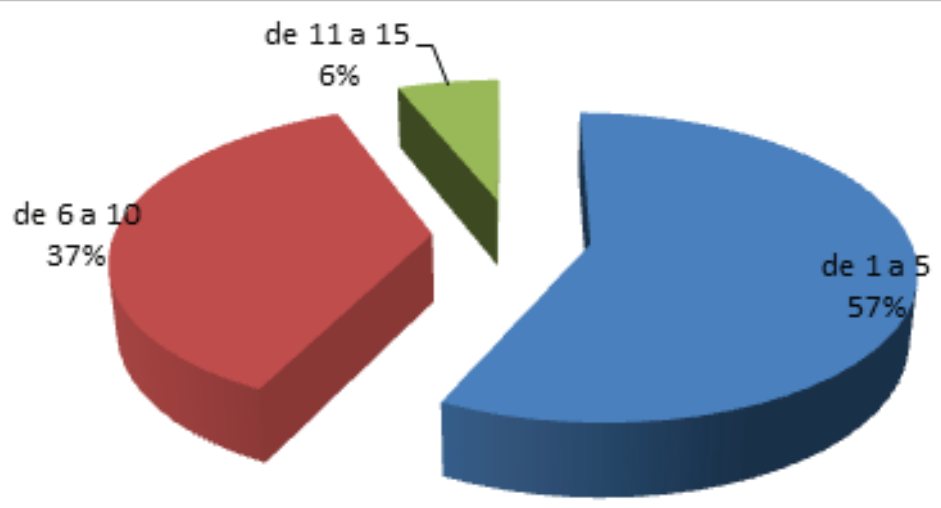

Fuente: Elaboración propia con base en los resultados de la entrevista a los productores.

Respecto al segundo indicador de importancia en materia de impactos, el nivel de ingreso de los beneficiarios del programa OP en la región III (cuadro 3), se encontró que de los 49 proyectos que siguen en operación, en $2 \%$ de ellos se ganaba menos de 500 pesos a la semana, en promedio (en el empleo que desempeñaba en su comunidad), antes de ser apoyado en la actividad productiva correspondiente, en $91.8 \%$ se ganaba de 500 a 1000 pesos a la semana y sólo el 6.1\% manifestó ganar más de 1000 pesos, en promedio, a la semana antes del apoyo. Después de recibir el financiamiento de OP para la actividad productiva unicamente el grupo que ganaba más de 1000 pesos antes del crédito mostró un cambio positivo en su capacidad de generación de ingresos, ya que se obtuvieron entre 2001 y 3000 pesos; lo que representa $14.3 \%$ y un aumento en su porcentaje de representación del número de personas, según nivel de ingreso, de 33\% en total. El grupo intermedio registró una reducción, en tanto que el primero de éstos no manifestó cambio alguno. Es decir, el grupo que ganaba de 500 a 1000 pesos, con el proyecto redujo su porcentaje a 83.7, esto como resultado directo de que el porcentaje de personas que ganaba más de 1 000 pesos se incrementó. 
Lo anterior se debe considerar como un ligero logro en el objetivo de mejorar los ingresos de los beneficiarios del programa y de sus familias. Y estos resultados podrían mejorarse si los productores tuvieran un mercado meta identificado para sus productos, una mejor calidad de los mismos y si realizaran reinversiones en las actividades productivas apoyadas.

\section{Cuadro 3}

Nivel de ingreso de los beneficiarios del programa $O P$

\begin{tabular}{|r|c|c|c|c|}
\hline $\begin{array}{c}\text { Antes de recibir el crédito su } \\
\text { ingreso era de: }\end{array}$ & $\begin{array}{c}\text { Total de personas } \\
\text { entrevistadas }\end{array}$ & Menos de 500 & $500-100$ & $1001-2000$ \\
\hline Número de personas & 49 & 1 & 45 & 3 \\
\hline Porcentaje & & 2.04 & 91.84 & 6.12 \\
\hline $\begin{array}{r}\text { Después de recibir el crédito su } \\
\text { ingreso es de: }\end{array}$ & & Menos de100 & $\begin{array}{c}100 \mathrm{a} \\
2000\end{array}$ & $2001-3000$ \\
\hline Número de personas & 49 & 1 & 41 & 7 \\
\hline Porcentaje & & 2.04 & 83.67 & 14.29 \\
\hline
\end{tabular}

Fuente: Elaboración propia con base en los resultados de la entrevista a los productores.

Si se parte de la información antes presentada, se puede decir que el programa OP sí generó empleos (temporales y permanentes) dado que para los beneficiados con sus apoyos sirvió para generar un buen número de fuentes de autoempleo y algunas de empleo, sin embargo, esto sólo se reflejó levemente, como se podrá observar más adelante en algunos de los indicadores del nivel de vida de ellos.

En lo que respecta a los indicadores del nivel de vida de los beneficiaros del programa $\mathrm{OP}$, en el parámetro de escolaridad se encontró que $25 \%$ tiene primaria incompleta, 16\% finalizó la educación primaria, 14\% manifestó que cursó parte de los estudios de secundaria, mientras que $25 \%$ cuenta con este nivel educativo y unicamente $16 \%$ de los beneficiados terminó los estudios de bachillerato o más (gráfica 2). Es de llamar la atención que al sumar el porcentaje de beneficiarios que no completó la primaria con los que no asistieron a la escuela, en esta región alcanzó un total de casi $30 \%$, lo cual hace evidente que este factor está influyendo en alguna medida en la administración y operación correcta de los proyectos de OP 
e impacta en el manejo y la reinversión de los ingresos obtenidos. Asimismo, se detectó que $79.6 \%$ de los beneficiarios tienen hijos que asisten a la escuela, desde el preescolar hasta la licenciatura, de los cuales $10.3 \%$ tienen hijos que están cursando una carrera universitaria; este último dato pone de relieve la conciencia que se va adquiriendo entre los habitantes del interior del estado sobre la importancia de proveer educación a los hijos para un mejor futuro.

\section{Gráfica 2}

Nivel de escolaridad de los beneficiarios apoyados

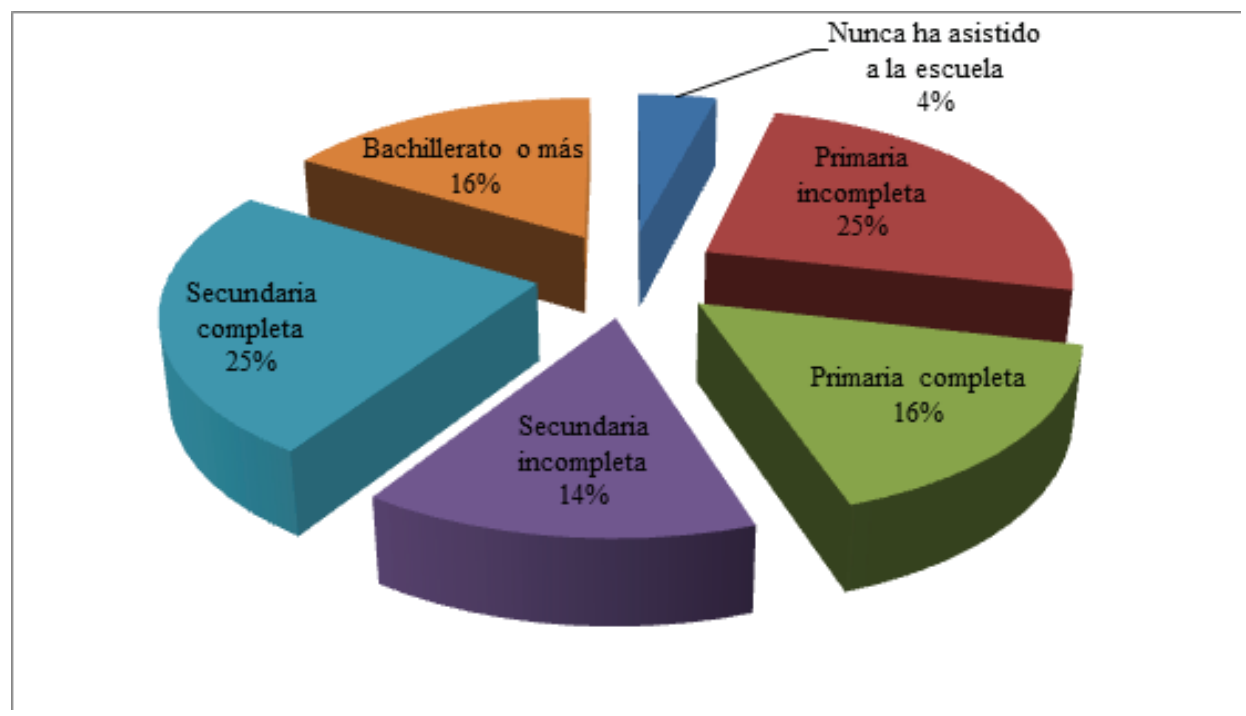

Fuente: Elaboración propia con base en los resultados de la entrevista a los productores.

Los otros indicadores evaluados en relación al nivel de bienestar presentaron las características que se relacionan en el cuadro 4 . 


\section{Cuadro 4}

Indicadores del nivel de vida de los beneficiarios de Opciones productivas

\begin{tabular}{|c|c|c|}
\hline Concepto & Antes del financiamiento & Después del financiamiento \\
\hline $\begin{array}{l}\text { Características de } \\
\text { la vivienda }\end{array}$ & $\begin{array}{l}4.1 \% \text { contaba con techos de } \\
\text { lámina de cartón, pared de } \\
\text { bajareque y piso de tierra } \\
26.5 \% \text { techo de lámina de } \\
\text { cartón o paja, pared de blocks } \\
\text { y piso de cemento } \\
69.4 \% \text { techo de concreto, } \\
\text { pared de blocks y piso de ce- } \\
\text { mento }\end{array}$ & $\begin{array}{l}0 \% \text { contaba con techos de lámi- } \\
\text { na de cartón, pared de bajareque } \\
\text { y piso de tierra } \\
20.4 \% \text { techo de lámina de cartón } \\
\text { o paja, pared de blocks y piso de } \\
\text { cemento } \\
79.6 \% \text { techo de concreto, pared } \\
\text { de blocks y piso de cemento }\end{array}$ \\
\hline $\begin{array}{l}\text { Disponibilidad de } \\
\text { servicio sanitario }\end{array}$ & $83.7 \%$ sí y $16.3 \%$ no & $87.7 \%$ sí y $12.2 \%$ no \\
\hline $\begin{array}{l}\text { Condiciones de sa- } \\
\text { lud }\end{array}$ & $\begin{array}{l}42.8 \% \text { sí contaba con servi- } \\
\text { cios de salud y } 57.1 \% \text { no. } \\
57.1 \% \text { recibía Seguro Social, } \\
\text { 9.5\% ISSSTE y } 33.3 \% \text { Segu- } \\
\text { ro Popular }\end{array}$ & $\begin{array}{l}87.7 \% \text { de los beneficiados tiene } \\
\text { acceso a servicios de salud y sólo } \\
\text { el } 12.2 \% \text { no tiene acceso. } \\
32.55 \% \text { de los beneficiados por } \\
\text { el Seguro Social, } 6.97 \% \text { por el } \\
\text { ISSSTE y } 60.5 \% \text {, por el Seguro } \\
\text { Popular }\end{array}$ \\
\hline $\begin{array}{l}\text { Tipo de alimenta- } \\
\text { ción }\end{array}$ & $\begin{array}{l}\text { 95.91\% consumía carne, fru- } \\
\text { tas, legumbres y leche } \\
4.09 \% \text { consumía frutas, le- } \\
\text { gumbres y leche como com- } \\
\text { plemento } \\
\text { Una vez por semana }\end{array}$ & $\begin{array}{l}95.91 \% \text { consumía carne, frutas, } \\
\text { legumbres y leche } \\
4.09 \% \text { consumía frutas, legum- } \\
\text { bres y leche como complemento } \\
\text { Una a tres veces por semana }\end{array}$ \\
\hline $\begin{array}{l}\text { Adquisición de } \\
\text { equipo electrodo- } \\
\text { méstico }\end{array}$ & $0 \%$ & $5 \%$ sí \\
\hline
\end{tabular}

Fuente: Elaboración propia con base en los resultados de la entrevista a productores. 
Es importante señalar que, entre los beneficiarios del programa OP en la región III de Yucatán, la recepción de otro tipo de apoyo por parte del gobierno federal o estatal se obtuvo en $44.89 \%$ de ellos, mientras que para $55.11 \%$ no. De los beneficiarios que tuvieron otros apoyos (gráfica 3), $27.27 \%$ lo recibieron en forma de pisos para su vivienda, a $27.27 \%$ se les construyó un pie de casa, a 18.18\% los apoyó el programa Oportunidades, a $9.09 \%$ se les ayudó con un baño en su hogar y a 9.09\% se les puso techo a sus viviendas.

\section{Gráfica 3}

Otros apoyos recibido por los beneficiarios

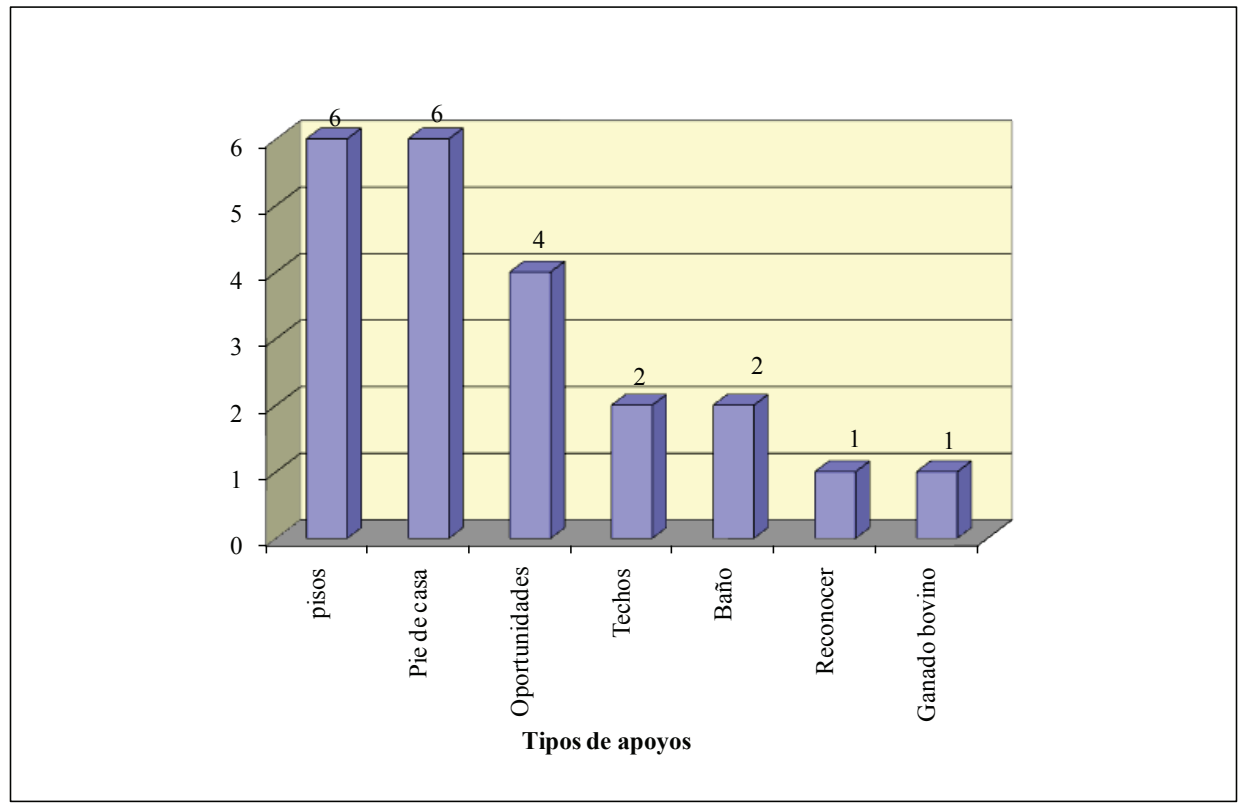

Fuente: Elaboración propia con base en los resultados de la entrevista a los productores.

Cabe aclarar también que la condición de acceso a servicios de salud mejoró más por la campaña de afiliación al seguro popular en los municipios estudiados, que como resultado directo de los apoyos del Programa en la región, ya que actualmente $60.46 \%$ de los beneficiarios que manifestaron tener acceso a servicios salud es por su registro en el seguro popular, el cual es gratuito. 
Por lo tanto, con base en los resultados anteriores y al considerar los principios que establecen la teorías relacionadas con el desarrollo económico, - que señalan como un factor importante para el desarrollo económico de una región que las personas accedan a mejores niveles de educación, salud, alimentación y de vivienda-, se puede decir que la puesta en marcha de los proyectos financiados por el programa OP en la región III de Yucatán, en general, no denotó mejoras en las condiciones de vida de la población; debido a que los cambios para muchos de los beneficiarios, que manifestaron mejoras en salud y vivienda, se debieron a otros apoyos otorgados por el gobierno federal o estatal, y no directamente como resultado del programa Opciones productivas en particular.

\subsection{Características de la gestión del programa OP en la región III de Yucatán}

En cuanto a las cinco características consideradas para evaluar la gestión del programa OP, se observó que en la primera, tiempo de ingreso y recepción del financiamiento, $93.9 \%$ de los beneficiarios manifestó que recibió el crédito de manera oportuna, mientras que una parte muy pequeña $(6.1 \%$ restante) recibió el apoyo en un periodo de seis meses posterior a la entrega de su solicitud.

En relación con la operación y administración del proyecto apoyado, $77.6 \%$ de los representantes indicaron que en esta característica no tuvieron problemas, no así $22.5 \%$ de ellos cuyos problemas más importantes fueron: a) desintegración del grupo, b) el grupo no devuelve el apoyo (los integrantes no lo están pagando y solamente paga la o el representante), c) se tiene alta mortalidad de los animales debido a falta de asesoría y capacitación y d) dificultad para adquirir los alimentos para los animales, debido a que el precio se ha elevado y, por ello, requieren de más capital para consolidar de mejor forma la actividad.

La situación descrita anteriormente la complementa la opinión de los funcionarios relacionados con la operación o gestión del programa en la entidad, quienes citaron que los problemas más importantes al respecto son: a) existencia de una elevada demanda de apoyos, en comparación con la capacidad de atención por parte de la Secretaría de Política Comunitaria 
y Social (SPCS), b) la asignación de los recursos no siempre se realiza de manera objetiva, c) se piden demasiados documentos al momento de registrar la solicitud vía internet en la página de la Secretaria de Desarrollo Social (Sedesol) federal, lo que hace más burocrático el proceso en lugar de simplificarlo, d) falta de acuerdo directo entre los funcionarios de primer nivel para la operación de programa, e) la respuesta de las solicitudes para con los beneficiarios es tardada y f) la página presenta muchos problemas de saturación, lo que genera atrasos en la captura de las solicitudes. Esto resulta contrastante e indica que el problema en la gestión de OP proviene, principalmente, de los encargados de administrar y operar el mismo, y no de los beneficiados.

Respecto a la tercera característica, que se relaciona con el número de veces que personal de la Sedesol y de la Secretaría de Política Comunitaria y Social realizó visitas de seguimiento al proyecto apoyado por el programa durante el tiempo en el que ha estado operando, $81.6 \%$ de los productores beneficiados señalaron que no han recibido la visita del personal de la Secretaría de Política Comunitaria, con respecto a la Sedesol federal dicha afirmación fue de 69.4 por ciento. Esta falta de atención a los productores beneficiados ha incidido en el seguimiento y operación del proyecto. Solamente $30.6 \%$ de los beneficiarios manifestó que recibieron visitas de seguimiento por parte del personal de la Sedesol, destacaron que se hacían con frecuencia de una a dos veces por año; para $18.4 \%$ de los que fueron visitados por la Secretaría de Política Comunitaria y Social la frecuencia también fue de una a dos veces por año.

Esta característica, junto con lo expresado con respecto a la capacitación de los beneficiarios de OP, permite deducir desde la perspectiva de la teoría del empleo, — que plantea la necesidad de una capacitación y asesoría para mejorar el rendimiento y productividad-, que resultó muy limitada en el programa, por lo que se puede afirmar que, la falta de seguimiento, es el factor que ha impedido que permanezcan más proyectos en operación en la región estudiada. De realizarse adecuadamente dicho seguimiento y conjugarlo con otros factores, como la búsqueda de mercados para sus productos, estos proyectos productivos podrían incidir, aún más, sobre la mejora de las condiciones de vida de las familias que se beneficiaron con los apoyos del programa OP en esta región, que es lo que se pretende, es 
decir, que la actividad productiva desarrollada en estas comunidades rurales sea una fuente de sustento familiar digno.

Con referencia a la cuarta característica, que tiene relación con los problemas de seguimiento y supervisión del proyecto apoyado, para 93.9\% de los beneficiados que recibieron la visita del personal manifestaron que no hubo ningún problema de esta índole, lo cual resulta entendible dada la poca frecuencia con la que realizaron, las distintas instancias encargadas, tal actividad. Por el contrario, únicamente $6.1 \%$ de los beneficiarios presentó algún problema al respecto, destacan: la variación constante en el precio del producto y la poca disponibilidad de algunos integrantes de los grupos de trabajo por desarrollar sus actividades.

En lo que concierne a la recepción de otro tipo de apoyo otorgado por parte del gobierno federal o estatal a los beneficiarios del programa (quinta característica), se tiene que $44.9 \%$ de ellos lo han recibido, mientras que para la mayoría de los beneficiarios no fue así. De los beneficiarios que recibieron otros apoyos se pudo constatar que entre la ayuda adicional destacan: pisos para vivienda $(27.3 \%)$, construcción de pie de casa (27.3\%), Oportunidades (18.2\%), construcción de baño $(9.1 \%)$ y techo para vivienda $(9.1 \%)$.

Al contrastar los resultados obtenidos con las teorías relacionadas con la instrumentación de las políticas públicas, bajo la del enfoque Topdown, se observa que el aspecto burocrático es un factor que está generando frenos a la operación del programa OP en Yucatán, al alargar los tiempos de atención a las solicitudes de crédito (logro de objetivos del programa a través del tiempo), así como por la falta de acuerdo entre los funcionarios de primer nivel (comportamiento de los burócratas). Asimismo, desde el punto de vista de teoría de la participación burocrática y la organización, se tiene que entre los problemas que existen en la gestión del programa en la entidad son la demora en la respuesta a los beneficiarios sobre si procede o no su proyecto (proceso de comunicación), así como la falta de acuerdo directo entre los funcionarios de primer nivel para la operación del programa (capacidades y actitudes hacia el programa): lo cual evidentemente impide su realización, ya que no se concretan los convenios establecidos y se reduce el impacto del programa. Sin embargo, esto no es exclusivo del programa en cuestión, ya que es una condición común en los 
programas gubernamentales que ofrecen alternativas de desarrollo a las familias de bajos recursos en el país. Es también de importancia señalar que en la organización de OP se observó la carencia de una fecha de apertura y cierre de ventanilla en la secretaría responsable de su operación, lo que ocasiona una gran cantidad de solicitudes de apoyo que difícilmente se podrán atender, debido a que los recursos son cada vez más limitados, y abona con ello a la problemática de burocracia en la operación del mismo.

\subsection{Indicadores de eficacia en la gestión del programa OP en la región III de Yucatán}

El papel que desempeña en la actualidad la Secretaría de Política Comunitaria en la gestión del programa Opciones productivas en su vertiente de cofinanciamiento, consiste en su ejecución mediante un convenio que firma con la delegación de la Sedesol Federal, y abarca desde la recepción de las solicitudes hasta la realización del pago del crédito otorgado a los beneficiarios. El mecanismo que emplea la Secretaría para seleccionar a los sujetos de crédito contempla, principalmente, una revisión documental para verificar que se cumple con los requisitos básicos del programa y la existencia de un comité de validación integrado por representantes del sector empresarial, educativo y gubernamental, el cual determina cuales son los proyectos susceptibles de recibir el apoyo.

La Secretaría de Política Comunitaria en su operación del programa OP presenta hoy en día problemas en cuanto al porcentaje de atención de las solicitudes que reciben, principalmente porque los recursos financieros con que cuenta para tal acción resultan insuficientes para atender la alta demanda que se tiene del programa, ya que ni la delegación de Sedesol ni el gobierno estatal tienen los suficientes recursos para realizar la aportación que les corresponde, por ejemplo, en la Secretaría de Política Comunitaria y Social de Yucatán sólo se atiende entre 5 y $10 \%$ de las solicitudes recibidas anualmente. Otra área problemática se registra en cuanto a la división de las tareas para cada actor del programa, si bien teóricamente son adecuadas, en las reglas de operación se estipulan de manera detallada, no obstante, en la operación muchas veces no se respeta esta división entre los beneficiarios del programa. Por ejemplo, la delegación de la Sedesol 
debería ser sólo la entidad normativa, sin embargo, también ejecuta; en contraste, la SPCS, que es la institución ejecutora, en ocasiones no realiza esta función por qué no se firman los convenios correspondientes. Como resultado los beneficiarios, en ocasiones, no cumplen con la recuperación del crédito o no invierten en las actividades para las que lo solicitaron.

Otro problema en la eficacia de la gestión se relaciona con los medios de promoción y difusión del programa que sirven para dar a conocerlo entre las personas interesadas, ya que, si bien para una parte de los gestores de OP si son adecuados en la actualidad, para otros no, los cuales consideran que el programa no tiene difusión en los medios de comunicación masiva de la entidad, como la televisión y radio. Esto último, coincide con lo expuesto por los beneficiarios del programa en la región III estudiada, quienes en $73 \%$ de los casos se enteraron de éste por medio del ayuntamiento; el problema es que no lo hacen extensivo sino que sólo lo comunican a unas cuantas amistades o conocidos.

Otro aspecto negativo en la gestión de OP es respecto al incumplimiento de los objetivos planteados en el programa, aquí hubo divergencia en las opiniones de sus administradores, ya que para algunos los objetivos no se están cumpliendo básicamente porque: a) los que reciben el crédito no siempre cumplen con los criterios de elegibilidad marcados por el programa, b) no existe en muchos casos reinversión y c) no existe seguimiento y acompañamiento de los proyectos apoyados. Para otros, en cambio, los objetivos planteados en el programa se cumplen principalmente porque: a) los beneficiarios realizan las actividades productivas que solicitan, b) se genera autoempleo entre los beneficiarios y c) se contribuye a la generación de ingresos para las familias apoyadas. Esto coincide con la perspectiva de los beneficiarios expuesta anteriormente, ya que, como se indicó, únicamente $52 \%$ de los proyectos apoyados continúan en operación, y generan autoempleo en la mayoría de los casos. Sin embargo, por la carencia de seguimiento de los mismos se redujo el éxito de más proyectos en la región estudiada.

En lo que respecta al impacto en la generación de empleos en los municipios donde se está aplicando el programa, los administradores del mismo coinciden en forma afirmativa, aunque sólo sea en la modalidad de autoempleo, hecho que concuerda con lo expresado por los beneficiarios de la región III. 
En cuanto a los aciertos que ha tenido el programa Opciones productivas en su gestión en el estado, los más importantes en opinión de sus administradores son: a) reglas de operación claras, b) el esquema de financiamiento involucra a más de una dependencia, c) son fáciles y pocos los requisitos para los solicitantes, d) la existencia de mecanismos de recuperación externos al gobierno y e) se orienta a las capacidades productivas de los beneficiarios.

En relación con los aciertos que ha tenido Opciones productivas en su vertiente de cofinanciamiento, respecto a su seguimiento existen divergencias, ya que algunos funcionarios afirman que el seguimiento que se le ha dado al programa en la entidad ha motivado a los productores beneficiados a ser más cumplidos en la actividad apoyada y a la devolución del crédito recibido; mientras que para otros no se le ha dado un seguimiento real y ejemplifican esto con el hecho de que, si bien una forma de seguimiento que se observa en la actualidad en OP es la recuperación económica del crédito, indicador del éxito económico del programa-, éste en la práctica no resulta ser suficiente de que el proyecto esté en funcionamiento, ya que es práctica común, entre los productores beneficiados, pagar el crédito recibido en su totalidad para que las instancias correspondientes les reembolsen un nuevo crédito, sin que se esté desarrollando la actividad productiva apoyada.

En cuanto a las deficiencias más importantes del programa en Yucatán en relación con su seguimiento, en opinión de los gestores éstas son: a) lejanía de las comunidades en donde se encuentran los beneficiarios, b) insuficiente presupuesto anual para dicha tarea, c) no se cuenta por parte de la SPCS con un programa propio para dar seguimiento a los proyectos o grupos de beneficiados y d) no se aprovecha la red de mentores que promueve la Sedesol federal.

Por último, en lo que concierne a la forma de operación actual del programa y lo que se le podría modificar para mejorarlo, para sus gestores lo más importante sería:

- Dado que es un programa federal y que las reglas de operación se emiten desde las oficinas centrales, en la práctica se debería de cumplir en forma estricta, es decir, recibir solamente las solicitudes que cumplan con estricto apego las reglas de operación estipuladas. 
- Que en la SPCS se establezcan ventanillas con fechas de recepción de las solicitudes oportunas en tiempo.

- Despolitizar el programa y operarlo en función de sus objetivos productivos, económicos y sociales. Los funcionarios, tanto de la delegación de Sedesol como de la SPCS, deben dejar de lado las cuestiones políticas y centrarse en el objetivo de OP, que es el de ser una fuente de financiamiento para las personas con proyectos productivos en las comunidades de alta y muy alta marginación del estado, como en la zona analizada, para desarrollar capacidades y, sobre todo, ser fuente de ingresos que permitan acceder a mejores niveles de vida a estos grupos sociales.

- Hacer que los habitantes en condiciones de pobreza de otros municipios (como Kanasín, Ticul y Progreso), que no son sujetos de apoyo en la actualidad por OP, tengan acceso al mismo a través del comité de validación antes descrito, considerándolos como casos extraordinarios de acuerdo con la condición de la persona que solicite el crédito, ${ }^{1}$ o bien que se propongan adecuaciones a las reglas de operación con base en los grados de marginación de los municipios de la entidad, en donde también, a través de una verificación directa de los solicitantes, se constate su condición socioeconómica.

\section{CONCLUSIONES}

Con base en los resultados obtenidos se puede afirmar que el programa Opciones productivas, en su vertiente de cofinanciamiento, ha tenido un éxito parcial en esta región de la entidad, sin embargo, este nivel de éxito debería de ser más alto para que contribuya de manera directa y en forma relevante a la generación de empleos en esta región, una de las más marginadas del estado, y con esto se evite la migración de sus habitantes hacia Mérida, otros estados o al extranjero.

\footnotetext{
${ }^{1}$ La visita directa a campo, en donde se recabe la información socioeconómica del solicitante, deberá ser razón suficiente para justificar el por qué se otorgó el crédito a la persona, aun cuando el municipio en donde resida no esté considerado en las reglas de operación del programa.
} 
La permanencia de los proyectos en esta región contribuyó con $80 \%$ del autoempleo generado entre los integrantes de los grupos apoyados por el programa, en términos de valores absolutos esto significa que en la región estudiada se generaron 365 empleos como resultado del programa.

Por otra parte, el programa sólo está mejorando el nivel de ingreso de unos cuantos de sus beneficiarios en los municipios de esta región y, en general, no está contribuyendo a mejorar la calidad de vida de estas personas, esto último ha sido más bien resultado de otros apoyos otorgados por el gobierno federal o estatal.

La problemática que existe en la gestión del programa se circunscribe principalmente a: i) excesiva demanda de solicitudes de apoyo en la Secretaría de Política Comunitaria y Social, ii) asignación de recursos no siempre objetiva, iii) retraso en la respuesta a los beneficiarios, iv) seguimiento de proyectos no adecuado y v) conflictos de coordinación que no permiten la firma de acuerdos de colaboración entre la Sedesol y la SPCS.

Por último, se recomienda para corregir las deficiencias observadas: i) que las dependencias, normativa y operativa del programa, den seguimiento puntual a los proyectos (presupuestar recursos para dar seguimiento y supervisión) y, sobre todo, se brinde capacitación a los productores (planificar e incluir en las reglas de operación la capacitación técnica); ii) que la SPCS establezca convenios con tiendas o empresas regionales y del estado para la compra de productos procedentes de los grupos financiados, es decir, crear mercado y consolidar las actividades apoyadas y iii) que el comité técnico de validación sea un órgano colegiado que filtre los proyectos más aptos para recibir el crédito al considerar: rendimiento económico, impacto social, grupos que lo presentan, condición del municipio de donde proviene, entre otros criterios. 


\section{BIBLIOGRAFÍA}

Altimir, O. 2003. La dimensión de la pobreza en América Latina, Cuadernos de la CEPAL, Santiago de Chile.

Campos, Ma. Lorena y C. Pasquali. 2010. "Evaluación de la gestión de programas de reciclaje en escuelas de educación básica", Omnia, 16(1): 140-158.

Coneval. 2007. Evaluación de la implementación del programa Opciones Productivas, México.

DOF. 2010. Reglas de Operación del Programa de Opciones Productivas para el ejercicio fiscal 2010, México, Secretaría de Gobernación, en: http://dof.gob.mx/nota_detalle.php?codigo $=5173568 \&$ fecha $=31 / 12 / 2010$.

Elmore, R. 1978. "Organizational models of social program implementation”, Public Policy, 26: 185-228.

Hernández Sampieri, R., C. Fernández Collado y P. Baptista. 2010. Metodología de la investigación, México, McGraw Hill.

Kaufman, Herbert. 1973. Administrative Feedback: monitoring Subordinates behaviour. Washington, D.C., Brookings Institution.

Keynes, J. 1972. Teoría general sobre el empleo, el interés y el dinero, Madrid, Biblioteca de economía, Ediciones Orbis.

Lacalle Calderón, M.,S. Rico Garrido y J. Durán Navarro. 2008. "Estudio piloto de evaluación de impacto del programa de microcréditos de Cruz Roja Española en Ruanda”, Revista de Economía Mundial,19: 83-104.

Martínez Villa, Claudio. 2007. Diagnóstico y evaluación de la gestión del programa Chile Barrio,, Estudios de caso núm. 98,,Magíster en Gestión y Políticas Públicas, Universidad de Chile, en: http:// www.mgpp.cl/estudios_de_caso/diagnostico-y-evaluacion-de-lagestion-del-programa-chile-barrio/.

Osuna, J. y C. Márquez. 2005. Guía para la evaluación de políticas públicas, Instituto de Desarrollo Regional, España.

Scott, J. 2006. "Pobreza rural y políticas públicas", ponencia presentada en el Seminario desarrollo rural y el sector agroalimentario, falta lugar. 
SHCP. 2007. Sistema de evaluación del desempeño, junio, México, Gobierno Federal.

So, Alvin Y. 1990. Social change and development. Modernization, dependency and world system theories, Londres, SAGE Library of Social Research 178. Taylor, Yúnez y González (2007), Estudios sobre Políticas Públicas para el Sector Rural en México, Informe Consolidado de resultados del Proyecto Políticas y Gasto Público Federal en el Sector Rural en México para el Banco Interamericano de Desarrollo. En: www.cedrssa.gob.mx/includes/asp/download.asp?iddocumento...

Yúnez, Antonio. 2010. Las reformas al agro mexicano y algunos de sus efectos, en: http://puertacee.wordpress.com/2010/03/04/las-reformas-al-agro-mexicano-y-algunos-de-sus-efectos/. 